\title{
SPECTRAL ANALYSIS OF THE DIRAC OPERATOR ON A 3-SPHERE
}

\author{
YAN-Long FAnG, Michael LeVITIN AND DMitri VASSILIEV
}

\begin{abstract}
We study the (massless) Dirac operator on a 3-sphere equipped with Riemannian metric. For the standard metric the spectrum is known. In particular, the eigenvalues closest to zero are the two double eigenvalues $+3 / 2$ and $-3 / 2$. Our aim is to analyse the behaviour of eigenvalues when the metric is perturbed in an arbitrary smooth fashion from the standard one. We derive explicit perturbation formulae for the two eigenvalues closest to zero, taking account of the second variations. Note that these eigenvalues remain double eigenvalues under perturbations of the metric: they cannot split because of a particular symmetry of the Dirac operator in dimension three (it commutes with the antilinear operator of charge conjugation). Our perturbation formulae show that in the first approximation our two eigenvalues maintain symmetry about zero and are completely determined by the increment of Riemannian volume. Spectral asymmetry is observed only in the second approximation of the perturbation process. As an example we consider a special family of metrics, the so-called generalized Berger spheres, for which the eigenvalues can be evaluated explicitly.
\end{abstract}

Mathematics subject classification (2010): 35Q41, 35P15, 58J50, 53C25.

Keywords and phrases: Dirac operator, spectral asymmetry, generalized Berger spheres.

\section{REFERENCES}

[1] M. F. Atiyah, V. K. Patodi And I. M. Singer, Spectral asymmetry and Riemannian geometry, Bull. London Math. Soc. 5 (1973), 229-234.

[2] M. F. ATiYAh, V. K. PATOdi And I. M. Singer, Spectral asymmetry and Riemannian geometry I, Math. Proc. Camb. Phil. Soc. 77 (1975), 43-69.

[3] M. F. Atiyah, V. K. Patodi And I. M. Singer, Spectral asymmetry and Riemannian geometry II, Math. Proc. Camb. Phil. Soc. 78 (1975), 405-432.

[4] M. F. ATIYAh, V. K. Patodi AND I. M. Singer, Spectral asymmetry and Riemannian geometry III, Math. Proc. Camb. Phil. Soc. 79 (1976), 71-99.

[5] Z. AVETISYAN, Y.-L. FANG AND D. VASSILIEV, Spectral asymptotics for first order systems, Journal of Spectral Theory 6 (2016), 695-715.

[6] Z. Avetis yan, Y.-L. Fang, N. Saveliev and D. Vassiliev, Analytic definition of spin structure, Journal of Mathematical Physics 58 (2017), 082301.

[7] C. B ÄR, The Dirac operator on homogeneous spaces and its spectrum on 3-dimensional lens spaces, Archiv der Mathematik 59 (1992), 65-79.

[8] C. B ÄR, The Dirac operator on space forms of positive curvature, Journal of the Mathematical Society of Japan 48 (1996), 69-83.

[9] C. B ̈̈R, Metrics with harmonic spinors, Geometric and Functional Analysis 6 (1996), 899-942.

[10] C. B ÄR, Dependence of the Dirac spectrum on the spin structure, Séminaires et Congrès 4 (2000), $17-33$.

[11] J.-P. BourguignON AND P. GAUduChOn, Spineurs, opérateurs de dirac et variations de métriques, Communications in Mathematical Physics 144 (1992), 581-599.

[12] O. Chervova, R. J. Downes And D. VASsiliev, Spectral theoretic characterization of the massless Dirac operator, Journal of the LMS 89 (2014), 301-320.

[13] J. S. DOW KER, Vacuum energy in a squashed Einstein Universe, In Quantum theory of gravity: essays in honor of the sixtieth birthday of Bryce S. De Witt. Edited by Steven M. Christensen. Adam Hilger Ltd, Bristol, 1984, 103-124 
[14] J.S. Dow KeR AND D.F. PetTENGILL, The quantum mechanics of the ideal asymmetric top with spin, J. Phys. A: Mathematical, Nuclear and General 7 (1974), 1527-1536.

[15] R. J. Downes, M. LeVITIN AND D. VASSILIEV, Spectral asymmetry of the massless Dirac operator on a 3-torus, Journal of Mathematical Physics 54 (2013), 111503.

[16] R. J. Downes AND D. VASSILIEv, Spectral theoretic characterization of the massless Dirac action, Mathematika 62 (2016), 701-718.

[17] Y.-L. FANG, Analysis of first order systems on manifolds without boundary: a spectral theoretic approach, PhD thesis, University College London, 2017.

[18] Y.-L. FANG AND D. VASSILIEV, Analysis as a source of geometry: a non-geometric representation of the Dirac equation, J. Phys. A: Math. Theor. 48 (2015), 165203.

[19] Y.-L. FANG AND D. VASSILIEV, Analysis of first order systems of partial differential equations, In Complex Analysis and Dynamical Systems VI: Part 1: PDE, Differential Geometry, Radon Transform. AMS Contemporary Mathematics series 653 (2015), 163-176.

[20] C. Godbout, Chern-Simons classes and the Ricci flow on 3-manifolds, Proceedings of the AMS 141 (2013), 2467-2474.

[21] N. Hitchin, Harmonic spinors, Advances in Mathematics 14 (1974), 1-55.

[22] H. B. Lawson And M.-L. Michelsohn, Spin geometry, Princeton Mathematical Series, Princeton, 1989

[23] L. D. Landau And E. M. Lifshitz, Theory of elasticity, second English edition, translated by J. B. Sykes and W. H. Reid. Volume 7 of Course of theoretical physics. Pergamon Press, 1970.

[24] Yu.N. Obukhov, E.W. Mielke, J. Budczies, And F.W. Hehl, On the chiral anomaly in nonRiemannian spacetimes, Foundations of Physics 27 (1997) 1221-1236.

[25] Yu. Safarov And D. VASSILIEV, The asymptotic distribution of eigenvalues of partial differential operators, Amer. Math. Soc., Providence (RI), 1997, 1998.

[26] S. Sulanke, Berechnung des Spektrums des Quadrates des Dirac-Operators auf der Sphäre und Untersuchungen zum ersten Eigenwert von D auf 5-dimensionalen Räumen konstanter positiver Schnittkrümmung, Ph.D. thesis, Humboldt Universität zu Berlin, 1981.

[27] A. Trautman, The Dirac operator on hypersurfaces, Acta Physica Polonica B 26 (1995), 12831310. 